\title{
PESQUISA EM ENFERMAGEM: FATORES IMPLICADOS NA CATEGORIZAÇÃO DE DADOS *
}

\author{
Tânia Couto Machado Chianca ** \\ Telma Ribeiro Garcia**
}

CHIANCA, T.C.M.; GARCIA,T. R. Pesquisa em enfermagem: fatores implicados na categorização de dados. Rev.latino-am.enfermagem, Ribeirão Preto, v. 4, $\mathrm{n}^{\circ}$ especial, p. 173-83, abril 1996.

Neste estudo procuramos treinar uma enfermeira quanto ao emprego de técnicas de comunicação de modo a que se tornasse mais terapêutica em relações interpessoais sucessivas. Durante a pesquisa, detectamos problemas de categorização que foram também apontados e analisados.

UNITERMOS: categorização, técnicas de comunicação

\section{INTRODUÇÃO}

A pesquisa em enfermagem é considerada por muitos como a melhor forma de se obter conhecimento. No entanto, nem sempre a mesma, por si só, pode solucionar problemas. Além disso, os cientistas também podem cometer erros, que podem dar a impressão de que nenhum resultado benéfico foi alcançado.

* Trabalho desenvolvido na disciplina Análise Crítica dos Modelos de Interação em Enfermagem - Programa de Pós-Graduação em Enfermagem, Área Enfermagem Fundamental, Escola de Enfermagem de Ribeirão Preto da Universidade de São Paulo, sob a orientação de Prof $^{a}$ Dr $^{a}$ Emília Campos de Carvalho e da Profa Maria Márcia Bachion

** Doutorandas em Enfermagem, Programa Interunidades da Escola de Enfermagem de Ribeirão Preto da Universidade de São Paulo. Professora Assistente do Departamento de Enfermagem Básica da Escola de Enfermagem da Universidade Federal de Minas Gerais e Professora Adjunta do Departamento de Enfermagem de Saúde Pública e Psiquiátrica da CCS/UFPB, respectivamente 
Isto porque existem várias limitações quando se emprega o método científico, o qual, por sua vez, também não é isento de falibilidade. No entanto, cada pesquisa que se faz é adicionada a um corpo maior de conhecimentos que vão se acumulando (POLIT \& HUNGLER, 1991).

Para se formar um corpo de conhecimentos é necessário que as pesquisas elaboradas sejam de qualidade, o que depende de sua adequacidade, ou seja, de sua habilidade para responder adequadamente à questão elaborada, e da formulação clara e lógica do problema. Segundo BRINK \& WOOD (1978) a adequacidade da pesquisa depende da qualidade de seus procedimentos de medida, que são a validade e a confiabilidade. Tais aspectos determinarão a acurácia da pesquisa, o que significa que seus resultados foram obtidos através de instrumentos e processos isentos de erros sistemáticos.

FEHRING (1987) afirma que, freqüentemente, ao conduzir seus estudos, os pesquisadores mensuram conceitos ou variáveis de natureza abstrata. O autor considera que, para um estudo ser significativo, do ponto de vista científico, os instrumentos de medida das variáveis necessitam ser não somente válidos, como confiáveis, tornando-se necessário que o pesquisador forneça evidências dessas características do instrumento de pesquisa que utilizou.

Para KAPLAN (1975) uma medida válida é aquela que mede o que se propõe a medir, porém pode fazer isto mal. Logo, a validez "requer que a medida esteja relativamente livre de erro". O erro é a medida do insucesso daquilo que se pretendia. Os erros podem estar relacionados à sensibilidade (poder de discriminação) e à confiabilidade (variação entre resultados de medidas repetidas). Sendo as condições onde se realizam as medidas constantes, é "de particular importância o observador que realiza a medida, e isso especialmente no campo das ciências do comportamento" (KAPLAN, 1975). Segundo FEHRING (1987) uma medida é válida quando está bem fundamentada em seus princípios ou evidências, sendo capaz de resistir às críticas.

Para KAPLAN (1975) utilizamos juízes como "medida humana" onde "são feitas estimativas por um conjunto de observadores presumivelmente competentes e, depois, tomada como medida da grandeza em questão uma adequada combinação estatística daquelas estimativas". Segundo o autor a relação entre o objeto de estudo e outros observadores não é subjetiva, pois é estabelecida com outros seres humanos ou com o próprio observador. O importante é o controle, a sensibilidade e confiabilidade levadas a efeito pelos juízes.

Em nosso presente estudo procuramos treinar uma enfermeira quanto a técnicas de comunicação propostas por HAYS \& LARSON (1970), e nos deparamos com algumas dificuldades provenientes de fatores desconhecidos até então para nós, relativos à categorização das técnicas propostas pelos autores e sua validação.

Resolvemos então tecer algumas considerações, bem como discutir as dificuldades encontradas. 


\section{REFERENCIAL TEÓRICO}

ROGERS (1951) considera que a filosofia operacional básica do terapeuta, que não precisa ser a verbalizada, determina o tempo que o mesmo levará para se tornar competente. A filosofia operacional básica inclui atitudes diante do valor e importância do indivíduo e o respeito para com ele. A maneira como o terapeuta olha a pessoa é um processo contínuo de aprendizagem, desenvolvimento e integração pessoal. O autor afirma que o estudo profundo da conduta do terapeuta por ele mesmo realça, altera, modifica a atitude e a hipótese que tomará numa próxima entrevista com o cliente.

Quando ouvimos e transcrevemos entrevistas e as estudamos, a análise que fazemos depende de nosso sistema de valores e, aspecto que tem sido pouco discutido.

As enfermeiras cada vez mais se preocupam com a relação que estabelecem com seus clientes, seja por causa da ênfase maior dada nos currículos das escolas, durante a graduação, em ver a pessoa como um todo, integrada bio-psico-espiritualmente, seja pelas atividades burocráticas que desenvolvem, o que demanda grande parte de seu tempo e afasta-as da cabeceira dos pacientes.

Às enfermeiras competem atividades de promoção de saúde, porém poucas são preparadas adequadamente para essa função, que envolve relações terapêuticas - aquelas que se estabelecem entre duas ou mais pessoas que se respeitam e participam conscientemente da relação, num processo bem definido, com objetivos claros a serem alcançados.

As enfermeiras são levadas a considerar que devem estar perto dos pacientes, ouvi-los, interessar-se por eles, responder às suas perguntas e não aumentar a ansiedade deles, mas elas não são treinadas para isso. Elas são treinadas para supervisionar e guiar o paciente, numa atitude muitas vezes maternal, não sendo treinadas para saber estabelecer relações terapêuticas com clientes, embora reconheçam a importância dessas (HAYS \& LARSON, 1970; STEFANELLI, 1985; FERRAZ, 1991; STACCIARINI, 1991).

Segundo HAYS \& LARSON (1970) "para relacionar-se terapeuticamente com um paciente é necessário que a enfermeira compreenda seu papel e sua relação com a doença". Os autores, enfatizando a relação enfermeira-paciente, pesquisaram as várias técnicas utilizadas na relação, definiram-nas e apresentaram-nas com o objetivo de ajudar o pessoal de enfermagem a compreendê-las também. Dividiram as técnicas interpessoais em terapêuticas e não terapêuticas, fornecendo princípios para as enfermeiras aprenderem a interagir terapeuticamente com pacientes (Quadro 1). 
QUADRO 1 - TÉCNICAS TERAPÊUTICAS E NÃO TERAPÊUTICAS PROPOSTAS POR HAYS \& LARSON (1970)

\begin{tabular}{|l|l|}
\hline \multicolumn{1}{|c|}{ Técnicas terapêuticas } & \multicolumn{1}{c|}{ Técnicas não-terapêuticas } \\
\hline 01. utilizando o silêncio & 01. tranqüilizando \\
\hline 02. aceitando & 02. fornecendo aprovação \\
\hline 03. reconhecendo & 03. rejeitando \\
\hline 04. oferecendo-se & 04. desaprovando \\
\hline 05. dando abertura para outros assuntos & 05. concordando \\
\hline 06. oferecendo um guia geral & 06. discordando \\
\hline $\begin{array}{l}\text { 07. localizando o evento no tempo ou na } \\
\text { seqüência }\end{array}$ & 07. aconselhando \\
\hline 08. fazendo observações & 08. sondando \\
\hline $\begin{array}{l}\text { 09. incentivando a descrição de } \\
\text { percepções }\end{array}$ & 09. desafiando \\
\hline 10. encorajando comparações & 10. testando \\
\hline 11. repetindo & 11. defendendo \\
\hline 12. refletindo & 12. solicitando uma explicação \\
\hline 13. focalizando & $\begin{array}{l}\text { 13. indicando existência de uma força } \\
\text { externa }\end{array}$ \\
\hline 14. explorando & 14. subestimando sentimentos expressos \\
\hline 15. dando informação & 15. fazendo comentários estereotipados \\
\hline 16. clareando & 16. dando respostas literais \\
\hline 17. apresentando a realidade & 17. usando de negativas \\
\hline 18. falando as dúvidas & 18. interpretando \\
\hline 19. procurando validação consensual & 19. introduzindo tópicos não relacionados \\
\hline 20. verbalizando o implícito & \\
\hline 21. incentivando avaliação & \\
\hline $\begin{array}{l}\text { 22. procurando transferir para } \\
\text { sentimentos }\end{array}$ & \\
\hline 23. sugerindo colaboração & \\
\hline 24. resumindo & \\
\hline $\begin{array}{l}\text { 25. incentivando formulação de um plano de } \\
\text { ação }\end{array}$ & \\
\hline
\end{tabular}

Vários autores (HAYS \& LARSON, 1970; STEFANELLI, 1985; FERRAZ, 1991; STACCIARINI, 1991) têm estudado o modelo de comunicação terapêutica na enfermagem. às trabalhos efetuados por estes autores visam permitir uma análise do processo interpessoal.

A eficácia de uma entrevista usando o modelo de comunicação terapêutica depende das habilidades de comunicação da enfermeira em relação às necessidades dos pacientes (HEIN, 1980). A autora cita várias técnicas como a utilização do silêncio, táticas de clarificação e de apoio que, usadas juntas ou separadamente, tornam a entrevista mais eficaz. Uma entrevista será mais eficaz quanto mais ajudar o paciente a mostrar, elaborar e descrever seus pensamentos e sentimentos. 
SUDDEEN et al (1981) afirmam que a comunicação terapêutica "permite e incentiva uma reciprocidade ou total participação da enfermeira-cliente na relação". Por outro lado a comunicação não terapêutica impede a formação de um relacionamento efetivo. Os autores apresentam técnicas específicas que podem ser utilizadas para facilitar o desenvolvimento de habilidades de comunicação terapêutica como ouvir, usar do silêncio, estabelecer roteiros, dar abertura, reduzir distância física, expressar algo que o paciente diz de uma outra forma, refletir, procurar clarificar, procurar validação consensual, centralizar e sumarizar.

\section{TREINAMENTO DE UMA ENFERMEIRA QUANTO ÀS TÉCNICAS DE COMUNICAÇÃO PROPOSTAS POR HAYS \& LARSON (1970) - METODOLOGIA E ANÁLISE}

Baseadas na análise das técnicas terapêuticas e não terapêuticas em relações enfermeira-cliente, consideramos a categorização como fator de identificação do padrão de comunicação e as categorias das técnicas propostas por HAYS \& LARSON (1970) importantes, e que o uso deliberado das técnicas aumenta a congruência enfermeira-cliente. Porem existem dificuldades na categorização das técnicas utilizadas na pesquisa que ao nosso ver, devem ser apontadas para que possam ser utilizadas no ensino, pesquisa e assistência.

Procedemos a duas entrevistas que foram filmadas, transcritas, analisadas, categorizadas, validadas e comparadas. A situação selecionada para as entrevistas foi a de experiências de cirurgia vivenciada por clientes.

Para avaliar a porcentagem de concordância entre os observadores em relação à situação de experiências de cirurgia vivenciadas por clientes utilizamos a fórmula de cálculo indicada por BATISTA (1977):

número de concordâncias

$\overline{\text { número de concordâncias + número de discordâncias }}$ $x 100$

O Índice de Concordância é o acordo entre juiz e enfermeira quanto à categorização das técnicas utilizadas pela segunda.

$\mathrm{Na}$ primeira entrevista o índice de concordância foi de $82 \%$ e, na segunda entrevista, foi de $96 \%$. Os índices de concordância obtidos são satisfatórios de acordo com BATISTA (1977) e BATISTA \& MATOS (1984), que preconizam um índice de concordância de 70 a $80 \%$ para o término do treinamento e o início da coleta de dados. No nosso caso houve um aumento de concordância na segunda entrevista quando comparada com a primeira. 
A enfermeira, a partir da análise da primeira entrevista, conscientizou-se de que, devido a experiências prévias, já utilizava técnicas de comunicação terapêutica (86\%), com predomínio das técnicas de "aceitação" $(40,6 \%)$ e "exploração" $(23,3 \%)$ com a cliente em um total de $86(100 \%)$ emissões verbais. A enfermeira utilizou técnicas não-terapêuticas como "sondagem" (12\%), "desaprovação" (1\%), "rejeição" (1\%) e "interpretação" (1\%) (Quadros 2 e 3).

Mediante estudo acerca das técnicas terapêuticas e não terapêuticas a enfermeira elaborou uma proposta de mudança para a segunda entrevista onde procurou preparar o ambiente; apresentar-se; acolher inicialmente a pessoa; dizer o que desejava do cliente; tentar obter informações gerais; saber o que foi significativo na experiência; observar; escutar; oferecer perguntas-guias; levar o cliente a localizar eventos citados e perguntados; explorar e oferecer-se para ajudar; tirar dúvidas; informar; refletir; repetir; apresentar a realidade; usar o silêncio para estimular que o cliente prosseguisse; incentivar descrições de percepções, comparações, avaliações e sentimentos.

\section{QUADRO 2 - CONCORDÂNCIA ENTRE ENFERMEIRA E JUIZ ACERCA DAS} TÉCNICAS TERAPÊUTICAS UTILIZADAS

\begin{tabular}{|c|c|c|c|c|c|}
\hline \multirow[b]{2}{*}{ Técnicas Terapêuticas } & \multicolumn{2}{|c|}{ Primeira } & \multicolumn{2}{|c|}{ Segunda } & \multirow[t]{2}{*}{ Total } \\
\hline & $\mathbf{F}$ & IC & $\mathbf{F}$ & IC & \\
\hline 01. Reconhecendo & 2 & & 2 & & 4 \\
\hline 02. Dando informação & - & & 27 & $29,3 \%$ & 27 \\
\hline 03. Explorando & 20 & $23,3 \%$ & 5 & $5,4 \%$ & 25 \\
\hline 04. Aceitando & 35 & $40,6 \%$ & 18 & $19,6 \%$ & 53 \\
\hline 05. Incentivando descrição de percepções & 1 & & 2 & & 3 \\
\hline 06. Repetindo & 3 & & 3 & & 6 \\
\hline 07. Incentivando avaliação & 1 & & 3 & & 4 \\
\hline $\begin{array}{l}\text { 08. Incentivando a formulação de um plano } \\
\text { de ação }\end{array}$ & - & & 2 & & 2 \\
\hline 09. Utilizando o silêncio & - & & 1 & & 1 \\
\hline 10. Oferecendo-se & - & & 5 & & 5 \\
\hline 11. Dando abertura para outros assuntos & 1 & & 2 & & 3 \\
\hline 12. Refletindo & 5 & & 1 & & 6 \\
\hline 13. Fazendo observações & 1 & & - & & 1 \\
\hline 14. Oferecendo um guia geral & 1 & & - & & 1 \\
\hline 15. Localizando o evento no tempo & 3 & & 1 & & 4 \\
\hline 16. Sugerindo colaboração & - & & 1 & & 1 \\
\hline 17. Encorajando comparações & 1 & & - & & 1 \\
\hline TOTAL & 74 & $86,0 \%$ & 73 & $79,3 \%$ & 147 \\
\hline
\end{tabular}

$\mathrm{F}=$ freqüência das concordâncias

IC = índice de concordância =

número de concordâncias

número de concordâncias + número de discordâncias

* os IC não discriminados não foram considerados significativos 
$\mathrm{Na}$ segunda entrevista, embora em menor proporção do que na primeira entrevista, a enfermeira mostrou-se terapêutica (79,3\%) em 92 emissões verbais. Embora tenha utilizado mais categorias de técnicas não-terapêuticas $(20,7 \%)$ do que na primeira entrevista, com predominância de "interpretração", "sondagem", "tranqüilização" e "fornecimento de aprovação". Podemos explicar o fato pela situação que se apresentava no momento: como a cliente se mostrava interessada em retirar dúvidas, a enfermeira utilizou muito $(29,3 \%)$ a técnica terapêutica "fornecendo informações". Usou também maior número e diversidade de técnicas terapêuticas de "exploração" (5,4\%), "silêncio", "descrição de percepções" e "avaliações da experiência de cirurgia e da interação", "dando abertura para outros assuntos" e "oferecendo-se para ajudar" $(5,4 \%)$.

QUADRO 3 - CONCORDÂNCIA ENTRE ENFERMEIRA E JUIZ ACERCA DAS TÉCNICAS NÃO - TERAPÊUTICAS UTILIZADAS

\begin{tabular}{|c|c|c|c|c|c|c|}
\hline \multirow[b]{2}{*}{ Técnicas Terapêuticas } & \multirow[t]{2}{*}{ Entrevistas } & \multicolumn{2}{|c|}{ Primeira } & \multicolumn{2}{|c|}{ Segunda } & \multirow[t]{2}{*}{ Total } \\
\hline & & $\mathbf{F}$ & IC & $\mathbf{F}$ & IC & \\
\hline 01. Fornecendo aprovação & & - & & 3 & $3,3 \%$ & 3 \\
\hline 02. Solicitando Explicação & & - & & 1 & & 1 \\
\hline 03. Concordando & & - & & 2 & & 2 \\
\hline 04. Defendendo & & - & & 1 & & 1 \\
\hline 05. Testando & & - & & 1 & & 1 \\
\hline 06. Tranqüilizando & & - & & 3 & $3,3 \%$ & 3 \\
\hline 07. Interpretando & & 1 & $1 \%$ & 4 & $4,3 \%$ & 5 \\
\hline 08. Aconselhando & & - & & 1 & & 1 \\
\hline 09. Sondando & & 10 & $12 \%$ & 3 & $3,3 \%$ & 13 \\
\hline 10. Desaprovando & & 1 & $1 \%$ & - & & 1 \\
\hline 11. Rejeitando & & 1 & $1 \%$ & - & & 1 \\
\hline TOTAL & & 13 & $15,0 \%$ & 19 & $20,7 \%$ & 32 \\
\hline
\end{tabular}

$\mathrm{F}=$ freqüência das concordâncias

IC = índice de concordância =

número de concordâncias

número de concordâncias + número de discordâncias

A técnica não-terapêutica de "sondagem" foi muito utilizada (12\%), mostrando que a enfermeira questionou muito, dando inclusive uma conotação de "utilização" do entrevistado. Destacamos que se torna necessário nessas ocasiões, que o entrevistador se coloque no lugar do entrevistado para que se sinta menos ansioso e valorize a pessoa como é e não somente pelo que possa lhe oferecer (Quadro 3). 
Para a utilização das técnicas é necessário antes, um treinamento das habilidades de comunicação (escutar, observar, guiar a interação e outras). A aprendizagem é um processo contínuo e a enfermeira mostrou-se muito ansiosa em comunicar terapêuticamente na segunda entrevista. Tal fato leva-nos a afirmar que o padrão da enfermeira é o de aceitar, explorar e fornecer informações, que parecem ser uma característica de enfermeiras não treinadas a utilizar técnicas terapêuticas mais apropriadas para os diversos momentos de uma interação.

$\mathrm{Na}$ análise das duas entrevistas contamos com a participação de um juiz que, a partir do treinamento das técnicas propostas por HAYS \& LARSON (1970), validou a categorização feita pela pesquisadora. No entanto, durante o processo de validação nos conscientizamos das dificuldades apresentadas durante o processo de utilização das técnicas. É importante assinalar que consideramos validada a categorização quando ocorreu concordância de no mínimo $80 \%$ entre a observadora (enfermeira) e o juiz no emprego, pela primeira, das técnicas de comunicação.

Concordamos com KAPLAN (1975) que "quanto mais exatamente conhecidas nossas respostas, mais facilmente podem elas ser manipuladas" porém, o pesquisador deve estar atento aos sete fatores intrínsecos, apontados por DENZIN (1989), que fornecem vieses e distorções à validação.

O primeiro deles são os fatos que ocorrem entre uma categorização e outra. A familiaridade do pesquisador e do juiz com as técnicas propostas; as experiências prévias; as ocorrências entre a primeira e a segunda entrevista; as leituras que os mesmos fizeram com relação ao referencial, além das experiências pessoais das pessoas entrevistadas.

O segundo fator é a diferença de maturidade tanto do pesquisador quanto do juiz, pois as relações que estabelecem poderão levar a mudanças em um ou no outro. Tais mudanças são acumulativas e os resultados podem não ser os mesmos dos primeiros encontrados.

Em nosso estudo, entre a primeira e a segunda categorização, a enfermeira e o juiz travaram discussões, estudaram mais profundamente o modelo e amadureceram suas idéias acerca do referido modelo, o que pode ser responsável pelas mudanças no padrão da segunda categorização em relação à primeira.

O terceiro fator é a susceptibilidade a vieses, onde proposições são desenvolvidas para serem aplicadas a todas as pessoas sob análise. A tendência a ser favorável com a pessoa e concordar (ou discordar) com as proposições, freqüentemente afeta as opiniões emitidas. No nosso caso, por diversas vezes, o juiz afirmou "sentir-se obrigado" a utilizar as categorias propostas pelos autores do trabalho, mesmo que a seu ver, as categorias não se adequassem às unidades de fala.

O quarto fator consiste na troca de juizes durante o processo. Tal fato não aconteceu conosco, o que nos teria obrigado a iniciar novamente o processo, no intuito de garantir a validade de nossa análise.

O quinto fator contempla as reações da enfermeira. Como a mesma está envolvida no processo, deve anotar todas as modificações que for observando, para 
avaliar seu grau crescente ou decrescente em função da terapeuticidade. Depois da primeira entrevista estabelecemos uma proposta de mudança frente às modificações observadas de comportamento comunicativo.

O sexto fator evidencia as mudanças na enfermeira. Quanto mais entrevistas forem sendo feitas, categorizadas, validadas e analisadas, mais mudanças serão observadas e, conseqüentemente, mais treinada estará a enfermeira em estudo. É preciso ir anotando as mudanças para que não se corra o risco de perder a objetividade que vai se obtendo com o decorrer do processo.

O último fator congrega os aspectos peculiares das situações onde as categorias são discriminadas. São importantes as relações estabelecidas entre pesquisador e juiz, as regras que se estabelecem para os dois, o número e conteúdo das categorias a serem estudadas e analisadas, a liberdade para se considerar outras categorias, as maneiras de se registrar as diferenças. No nosso caso as categorias eram em número elevado e o conteúdo das mesmas deixava, às vezes, a desejar, dando margem à elaboração de novas possíveis categorias de técnicas não terapêuticas, como a de antecipação da enfermeira à fala do cliente e a de mudança temática pela enfermeira.

Em nosso estudo, também consideramos que o grande número e conteúdos das categorias analisadas interferiram negativamente na validação procedida. Cremos que, quanto menor o número de categorias, maior a fidedignidade das observações a serem feitas. O tempo necessário para a categorização é longo e pode determinar irregularidades.

Para que se garanta o rigor em pesquisas de tal natureza, ressaltamos a importância da enfermeira se familiarizar com os fatores acima apontados para o desenvolvimento de pesquisas que envolvem categorização de dados. Da mesma forma, é preciso que se ressalte a complexidade observada em utilizar referenciais de comunicação na pesquisa em enfermagem, em especial nos passos metodológicos, e a correspondência que se deve garantir entre os mesmos e o referencial teórico adotado.

\section{NURSING RESEARCH: FACTORS INVOLVED IN THE CATEGORIZATION OF DATA}

In this study we aimed at training a nurse to apply communication techniques in order to become more therapeutic in her successive interpersonal relations. Along the research we found categorization problems that were considered and analyzed.

UNITERMS: categorization, communication techniques

Rev. Latino-am.enfermagem - Ribeirão Preto - v. 4 - n. especial - p. 173-183 - abril 1996181 


\title{
PESQUISA EN ENFERMERÍA: FACTORES IMPLICADOS EN LA CATEGORIZACIÓN DE DATOS
}

\begin{abstract}
En este estudio procuramos entrenar una enfermera cuanto a lo empleo de técnicas de comunicación de modo a tornarse más terapéutica en relaciones interpersonales sucesivas. Durante la pesquisa percibimos problemas de categorización que fueran también apuntados y analizados.
\end{abstract}

UNITERMOS: categorización, técnicas de comunicación

\section{REFERÊNCIAS BIBLIOGRÁFICAS}

01. BATISTA, C.G. Concordância e fidedignidade na observação. Psicologia, v. 3, n. 2, p. 39-49, 1977.

02. BATISTA, C.G.; MATOS, M. A. O acordo entre observadores em situação de registro cursivo: definições e medidas. Psicologia, v. 3, n.10, p. 57-69, 1984.

03. BRINK, P. J.; WOOD, M. J. Basic steps in planning nursing research: from question to propose. Duxbury Press. Belmont, 1978.

04. DENZIN, N. K. The research act. A theoretical introduction to Sociological methods. $3^{\mathrm{a}}$ ed. Prentice Hall. New Jersey. 1989.

05. FEHRING, R.J. Methods to validate nursing diagnoses. Heart and Lung, v. 16, n. 6, p. 625-9, nov., 1987.

06. FERRAZ, A. F. Análise da comunicação enfermeira-paciente em hospital geral. São Paulo, 1991. 106p. Dissertação (Mestrado) - Escola de Enfermagem de São Paulo, Universidade de São Paulo.

07. HAYS, J. S.; LARSON, K. Interaction with patients. New York, McMillan, 1970. $282 p$.

08. HEIN, E. C. Communication in Nursing Practice. $2^{\mathrm{a}}$ ed. Little Brown. Boston, 1980.

09. KAPLAN, A. A conduta na pesquisa. Metodologia para as ciências do comportamento. EPU/EDUSP. São Paulo. 1975.

10. LITTLEJOHN, S. W. Fundamentos teóricos da comunicação humana. Rio de Janeiro: Guanabara. 1988.

11. POLIT, D. F.; HUNGLER, B. P. Nursing research. Principles and Methods. $4^{\mathrm{a}}$ ed. J. P. Lippincott Company. Philadelphia. 1991.

12. ROGERS, C. R. Terapia centrada no paciente. Martins Fonseca. Lisboa. 1951. 
13. STACCIARINI, J. M. R. Assistência ao paciente psiquiátrico. Análise da comunicação da enfermeira. Ribeirão Preto, 1991. 101 p. Dissertação (Mestrado) - Escola de Enfermagem de Ribeirão Preto, Universidade de São Paulo.

14. STEFANELLI, M. C. Ensino de técnicas de comunicação terapêutica enfermeira-paciente. São Paulo, 1985. Tese (Doutoramento) - Escola de Enfermagem, Universidade de São Paulo.

15. SUDDEN, S. J. et. al. Nurse-client interaction. $2^{\mathrm{a}}$ ed. St. Louis: Mosby Company, 1981. 